\title{
Vorteile für Enzalutamid beim Prostatakarzinom
}

\author{
Was ist die bessere Alternative bei \\ kastrationsrefraktärem Prostata- \\ karzinom - Enzalutamid oder Bicalu- \\ tamid? Daten aus zwei Phase-II-Stu \\ dien geben Hinweise.
}

\begin{abstract}
$\ddot{A}$ rzte um Celestia Higano aus Seattle prüften in der STRIVE-Studie bei 396 Männern, ob Enzalutamid im Vergleich zum noch häufig verwendeten $\mathrm{Bi}$ calutamid sowohl bei nicht metastasierten als auch metastasierten androgenunabhängigen Tumoren von Vorteil ist. Im Gegensatz zu Bicalutamid hat Enzalutamid keine partiell agonistische Aktivität an Androgenrezeptoren, zudem ist die Bindungsaffinität höher, deshalb versprechen sich die Forscher einen klinischen Vorteil.

257 Patienten in der Studie hatten Metastasen, keiner hatte zuvor eines der beiden Medikamente erhalten. Eine Hälfte bekam Enzalutamid (160 mg/d), die andere Bicalutamid $(50 \mathrm{mg} / \mathrm{d})$, die bisherige Androgendeprivation wurde beibehalten. Die Therapie erfolgte bis zum erneuten PSA-Anstieg oder bis zur radiologisch festgestellten Tumorpro-
\end{abstract}

gression. Im Median lag das progressionsfreie Überleben (PFS) mit Enzalutamid bei 19,4, mit Bicalutamid bei 5,7 Monaten. Männer mit metastasiertem Karzinom lebten unter Enzalutamid im Median noch 16,5 Monate progressionsfrei, mit Bicalutamid 5,5 Monate. Bei nicht metastasierten Tumoren betrug das mediane PFS unter Bicalutamid 8,6 Monate, unter Enzalutamid wurde der Medianwert im Studienverlauf nicht erreicht.

Eine radiologisch bestätigte Progression oder der Tod traten unter Enzalutamid zu $68 \%$ seltener bei metastasierten und zu $76 \%$ seltener bei nicht metastasierten Tumoren auf. Ein PSA-Anstieg wurde um $81 \%$ seltener festgestellt als unter Bicalutamid. Schwerwiegende unerwünschte Wirkungen waren in beiden Gruppen etwa vergleichbar häufig.

In ähnlicher Weise behandelten Forscher um Axel Heidenreich, Aachen, in der TERRAIN-Studie 375 Patienten mit androgenunabhängigem Prostatakarzinom. Alle hatten Metastasen, aber bislang keine oder nur geringe Symptome. $45 \%$ waren schon zuvor mit Bicalutamid behandelt worden. Die Männer erhielten entweder $160 \mathrm{mg}$ Enzalutamid oder $50 \mathrm{mg}$ Bicalutamid pro Tag. Hier betrug das mediane PFS unter Enzalutamid 15,7 Monate, unter Bicalutamid 5,8 Monate. Die mediane Zeit bis zur PSA-Progression lag bei 19,4 versus 5,8 Monaten.

Die Resultate waren also denen in STRIVE sehr ähnlich. Grad-3-Nebenwirkungen waren unter Enzalutamid allerdings etwas häufiger zu beobachten (31 vs. $23 \%$ ).

Fazit: Eine Behandlung mit dem Androgenrezeptorblocker Enzalutamid kann das PFS bei Patienten mit fortgeschrittenem Prostatatumor deutlich verlängern. Ihren Daten zufolge sollte eher Enzalutamid als Bicalutamid bei Männern mit wenig symptomatischen, androgenunabhängigen Tumoren verwendet werden, folgern die Forscher. Thomas Müller

Penson DF et al. Enzalutamide Versus Bicalutamide in Castration-Resistant Prostate Cancer: The STRIVE Trial. J Clin Oncol. 2016;doi:10.1200/ JCO.2015.64.9285.

Shore ND et al. Efficacy and safety of enzalutamide versus bicalutamide for patients with metastatic prostate cancer (TERRAIN): A randomised, double-blind, phase 2 study. Lancet Oncol. 2016;17(2):153-63.

\section{Hochrisiko-Prostatakarzinom: intensive Therapie zu toxisch}

\begin{abstract}
Langfristige Androgensuppression plus Bestrahlung ist der Standard für Patienten mit Hochrisiko-Prostatakarzinom. Nun wurde untersucht, ob Betroffene von einer Intensivierung mit zusätzlicher Chemotherapie profitieren.
\end{abstract}

\footnotetext{
n der Studie 9902 der Radiation Therapy Oncology Group (RTOG 9902) sollten Patienten mit Hochrisiko-Prostatakarzinom (PSA 20-100 ng/ml und Gleason-Score $\geq 7$ oder klinisches Stadium $\geq \mathrm{T} 2$ und Gleason-Score $\geq 8$ ) Bestrahlung und Androgensuppression mit oder ohne zusätzliche adjuvante Chemotherapie mit Paclitaxel, Estramustin und oralem Etoposid in vier 21-Tage-Zyklen erhalten, beginnend 28 Tage nach der Bestrahlung mit 70,2 Gy. Geplant war die Aufnahme von 1.440 Patienten, aber die Rekrutierung wurde wegen übermäBiger thromboembolischer Toxizität im
}

Chemotherapiearm nach nur 397 Patienten beendet.

Bei keinem der Endpunkte wurde ein signifikanter Unterschied zwischen beiden Gruppen (ohne vs. mit Chemotherapie) erreicht. Die 10-Jahres-Rate der randomisierten Patienten betrug für das Gesamtüberleben 65 versus $63 \%$ $(\mathrm{p}=0,81)$, für biochemisches Versagen 58 versus $54 \%(p=0,82)$, für eine lokale Progression 11 versus $7 \%(p=0,09)$, für Fernmetastasen 16 versus $14 \%(\mathrm{p}=0,42)$ und für das krankheitsfreie Überleben 22 versus $26 \%(p=0,61)$. Die häufigsten Ursachen für nicht prostatakrebsbedingte
Todesfälle waren kardiovaskuläre oder respiratorische Ereignisse $(50 \%)$ und Lungenkrebs (21\%). Anzumerken ist, dass wegen Toxizitäten und der vorzeitigen Beendigung der Studie 33,5\% der Patienten nur ein bis drei Zyklen und 25,5\% gar keine Chemotherapie erhielten.

Fazit: Bei Patienten mit Hochrisiko-Prostatakarzinom zeigt eine Chemotherapie zusätzlich zu Androgensuppression und Bestrahlung keine Vorteile, verursachte aber hohe Toxizität. Judith Neumaier

Rosenthal SA et al. A Phase 3 Trial of 2 Years of Androgen Suppression and Radiation Therapy With or Without Adjuvant Chemotherapy for High-Risk Prostate Cancer: Final Results of Radiation Therapy Oncology Group Phase 3 Randomized Trial NRG Oncology RTOG 9902. Int J Radiation Oncol Biol Phys. 2015;93(2):294-302. 